\title{
PREVALENCE OF TRYPANOSOMA CRUZI AND ORGAN ALTERATIONS IN VIRGINIA OPOSSUMS (DIDELPHIS VIRGINIANA) FROM WESTERN MEXICO - SHORT COMMUNICATION
}

\author{
Vincenzo CARnevali ${ }^{1}$, Benjamín Nogueda-TorRes ${ }^{2}$, María E. Villagrán-Herrera ${ }^{3}$, \\ José A. De Diego-CABrera ${ }^{4}$, Gonzalo ROCHA-CHÁvez ${ }^{5}$ \\ and José A. MARTÍNEZ-IBARRA ${ }^{6^{*}}$ \\ ${ }^{1}$ Department of Public Health and Infectious Diseases, University of Rome, Rome, Italy; \\ ${ }^{2}$ COFAA Grant Fellow, Laboratory of Helminthology, Department of Parasitology, \\ National School of Biological Sciences, National Polytechnic Institute, Mexico City, \\ Mexico; ${ }^{3}$ Department de Biomedical Research, School of Medicine, Autonomous \\ University of Queretaro, Santiago de Queretaro, Queretaro, Mexico; ${ }^{4}$ Department of \\ Preventive Medicine and Public Health, School of Medicine, Autonomous University of \\ Madrid, Madrid, Spain; ${ }^{5}$ Division of Health Sciences and ${ }^{6}$ Laboratory of Medical \\ Entomology, Department of Natural Sciences, Southern University Centre, \\ University of Guadalajara, 49000 Ciudad Guzman, Jalisco, Mexico
}

(Received 28 March 2017; accepted 6 November 2017)

Small populations of Virginia opossum (Didelphis virginiana) in western Mexico are endangered by hunting and natural predators as well as by different kinds of diseases. After two serological analyses using Serodia ${ }^{\circledR}$ latex particle agglutination and indirect haemagglutination (IHA) tests, 35 (53.03\%) of 66 collected opossums in two small towns in western Mexico were positive for the presence of Trypanosoma cruzi. Twenty-eight of the 35 seropositive opossums had pathological lesions: 11 had changes in only one organ, 13 in two organs, and four had pathological changes in three organs. Splenomegaly was the most common finding in the examined opossums, followed by hepatomegaly. These potentially fatal pathological changes could contribute to the scarcity of the opossum population, even leading to the extinction of this species in western Mexico.

Key words: Trypanosoma cruzi, Chagas disease, pathological changes, Didelphis, Mexico

Trypanosoma cruzi, the causative agent of Chagas disease, has been frequently reported in wildlife reservoir hosts. In Mexico, at least 30 animal species can be infected with $T$. cruzi, including humans and dogs (Velasco-Castrejón and Rivas-Sánchez, 2008). Many reservoir species have been reported from western Mexico, one of the most epidemiologically important areas for the zoonotic

"Corresponding author; E-mail: aibarra@cusur.udg.mx; Phone/Fax: 0052 (341) 575-2222 
transmission of $T$. cruzi in the country (Carabarin-Lima et al., 2013). Species of the genus Didelphis, reputed as one of the most important reservoir hosts for $T$. cruzi, are frequently in contact with human populations since they are ecological generalists; therefore, they are adaptive to a wide range of habitats and climates, including areas of urbanisation (Cruz-Salazar et al., 2014). Villagrán et al. (2011) reported some specimens of Virginia opossum (Didelphis virginiana) with pathological changes in the digestive tract (megacolon and megaoesophagus) from western Mexico. In many areas of Mexico, opossums are regarded as a threat for chickens and hens, because they invade human farms to feed on poultry. Since they also live in urban areas and look for food (e.g. stored corn and agave juice) in human backyards, many people consider them a threat, especially because they are aggressive when disturbed. Furthermore, in many areas of Mexico opossums are usually hunted and eaten by humans (Cruz-Salazar et al., 2014). In addition, since the average life expectancy of an opossum in the wild is 1.3 to 7 years, in many areas of western Mexico their populations can turn over in as little as 4.8 years, as it has been recorded in some areas of the United States of America (McManus, 1974). As a consequence, opossum populations are in danger of extinction in many areas of the Pacific coast of Mexico (Cruz-Salazar et al., 2014).

The aim of this study was to contribute to the knowledge on the effect of $T$. cruzi infection on the organs of Virginia opossums, and to estimate the probable impact of this infection on the survival rate of Virginia opossum populations in western Mexico.

In order to collect samples, Virginia opossums were searched in and around two villages (San Gabriel 20.10N, 103.40W and Apango, 19.50N, 103.35W) in a nearby geographic area of western Mexico to that area where inhabitants and vectors have been previously reported as infected by $T$. cruzi (Villagrán et al., 2011). Collections of Virginia opossums were carried out for two consecutive nights for 12 months along 2015, by using 20 live traps (Tomahawk Live Trap Co., Tomahawk, Wisconsin, USA). Traps were baited with a piece of bread covered with canned tuna fish conserved in oil (Villagrán et al., 2011). Traps were placed under trees at sunset and were checked at sunrise, since Virginia opossums have nocturnal feeding habits (Cruz-Salazar et al., 2014). Collected animals were transported to the laboratory (Southern University Centre, University of Guadalajara), where serological tests were performed in order to diagnose $T$. cruzi infection. Five to $10 \mathrm{ml}$ of blood was collected from each animal and the serum was separated. As serological tests, Serodia ${ }^{\circledR}$ (Latex Particle Agglutination, Fujirebio Diagnostics, Inc., Seguin, TX) (sensitivity of 100\%, specificity of 99.8\%) and indirect haemagglutination (IHA; Wiener Lab, Buenos Aires, Argentina) (sensitivity of $77.8 \%$, specificity of $99.8 \%$ ) were applied to evaluate the presence of anti-T. cruzi antibodies. In order to check the internal organs of the seropositive opossums for the presence of lesions associated with $T$. cruzi infection, they were anaesthetised with $0.25 \mathrm{~mL} / \mathrm{kg}$ ketamine (PISA Lab, Mexico) in- 
jected intramuscularly (SAGARPA, 1999; Plumb, 2010). Subsequently, two short $(2 \mathrm{~cm})$ incisions were carried out in the abdomen and chest areas of each specimen in order to endoscopically check its internal organs, by using an EG-45005 gastroscope (Fujifilm, Tokyo, Japan). All analyses included positive and negative controls with organ samples from previously studied opossums infected with T. cruzi, preserved in Alglifen (Castrejón-Calderón, 2014). After the endoscopic examination and closure of the incision, every animal was kept under surveillance in an individual cage for three days, following the maintenance and care regulations of the Norma Oficial Mexicana (SAGARPA, 1999). A serial number was tattooed on the right ear of each opossum as an identification sign, in order to avoid capturing and sampling the same individual twice. After the end of the post-surgery period, every Virginia opossum was returned to its original habitat and released.

Sixty-six Virginia opossums were collected during one year. Thirty-five $(53.03 \%)$ of them were seropositive for $T$. cruzi. The Serodia ${ }^{\circledR}$ test detected 35 seropositive opossums, whereas the IHA test only detected 32 .

Twenty-eight (80\%) of the 35 seropositive Virginia opossums had some anatomic/pathological changes: 11 specimens had alteration in one organ (cardiomegaly, megaoesophagus, hepatomegaly, megabladder), 13 had two affected organs and four had three affected organs (Table 1).

Table 1

Percentage of pathological changes in opossums infected by Trypanosoma cruzi in western Mexico

\begin{tabular}{lc}
\hline Pathological changes & $\begin{array}{c}\text { Affected opossums } \\
\mathrm{n}(\%)\end{array}$ \\
\hline Cardiomegaly & $3(10.7)$ \\
Megaoesophagus & $1(3.6)$ \\
Hepatomegaly & $1(3.6)$ \\
Megabladder & $1(3.6)$ \\
Splenomegaly & $5(17.9)$ \\
Splenomegaly + cardiomegaly & $7(25.0)$ \\
Splenomegaly + hepatomegaly & $6(21.4)$ \\
Splenomegaly + cardiomegaly + hepatomegaly & $4(14.2)$
\end{tabular}

The percentage of infected $D$. virginianus determined in the current study in western Mexico (50.3\%) was similar to the 52\% prevalence observed in $D$. virginianus from six southwestern and southern states of the USA (Brown et al., 2010 ), to the $52 \%$ prevalence of infection obtained in this species from Campeche, Mexico (Tamay-Segovia et al., 2017) and to the 53.9\% prevalence found in D. virginianus from southeastern Mexico (Ruiz-Piña and Cruz-Reyes, 2002), which confirms the importance of the Virginia opossum as a reservoir of T. cruzi (Velasco-Castrejón and Rivas-Sánchez, 2008) in different geographic areas. 
More than $60 \%$ of opossums infected by T. cruzi presented some kind of potentially fatal organ changes (Fidalgo-Álvarez et al., 2003). In conjunction with hunting by humans and depredation, these alterations might strongly contribute to the highly reduced opossum populations in western Mexico. Some complementary studies on factors affecting western Mexican populations of opossums are necessary for estimating the risk of reduction of those populations more accurately.

\section{Acknowledgements}

This research was supported by the 230128 UDG-CA-621 grant of the Secretaría de Educación Pública and by the SAC/CI/009/16 grant of the Universidad de Guadalajara.

\section{References}

Brown, E. L., Roeling, D. M., Gompper, M. E., Monello, R. J., Wenning, K. M., Gabriel, M. W. and Yabsley, J. (2010): Seroprevalence of Trypanosoma cruzi among eleven potential reservoir species from six states across the Southern United States. Vector Borne Zoonotic Dis. 10, 757-763.

Carabarin-Lima, A., González-Vázquez, M. C., Rodríguez-Morales, O., Baylón-Pacheco, L., Rosales-Encina, J. L., Reyes-López, P. A. and Arce-Fonseca, M. (2013): Chagas disease (American trypanosomiasis) in Mexico: An update. Acta Trop. 127, 126-135.

Castrejón-Calderón, T. (2014): Development of educational material: Hand and foot dissections preserved by plastination and Alglifen techniques [in Spanish]. FES Cuatitlán. Universidad Nacional Autónoma de México. 27 pp. Available from: avalon.cuautitlan2.unam.mx /biblioteca/tesis/852.pd (Accessed January 2017).

Cruz-Salazar, B., Ruiz-Montoya, L., Navarrete-Gutiérrez, D., Espinoza-Medinilla, E. E., VázquezDomínguez, E. and Vázquez, L. B. (2014): Genetic diversity and relative abundance of Didelphis marsupialis and Didelphis virginiana in Mexico [in Spanish]. Rev. Mex. Biodiv. 85, 251-261.

Fidalgo-Álvarez, L. E., Rojas-López, J., Ruiz de Gopegui-Fernández, R. and Ramos-Antón, J. J. (2003): Veterinary Medical Pathology [in Spanish]. Universidad de León, León, España. $622 \mathrm{pp}$.

McManus, J. J. (1974): Didelphis virginiana. Mamm. Species 40, 1-6.

Plumb, D. C. (2010): Manual of Veterinary Pharmacology [in Spanish]. Intermédica, Buenos Aires, Argentina. $1256 \mathrm{pp}$.

Ruiz-Piña, H. A. and Cruz-Reyes, A. (2002): The opossum Didelphis virginiana as a synanthropic reservoir of Trypanosoma cruzi in Dzidzilché, Yucatán, México. Mem. Inst. Oswaldo Cruz 97, 613-620.

SAGARPA - Secretaría de Agricultura, Ganadería, Desarrollo Rural, Pesca y Alimentación (1999): Norma Oficial Mexicana NOM-062-ZOO-1999 - Technical Specifications for Production, Maintenance and Use of Laboratory Animals [in Spanish]. Available from: www.fmvz.unam.mx/fmvz/principal/archivos/062ZOO.PDF (accessed January 2017).

Tamay-Segovia, P., Blum-Domínguez, S. C., Núñez-Oreza, L. A., Martínez-Hernández, F., GómezSolano, M. L., Maldonado-Vázquez, M. G. and Retana-Gulascón, Ó. G. (2017): Presence of Virginia opossum (D. virginiana) and pic (Triatoma dimidiata) infected with Trypanosoma cruzi in urban areas: preliminary evaluation in the city of Campeche, Mexico. Austral. J. Vet. Sci. 49, 35-38. 
Velasco-Castrejón, O. and Rivas-Sánchez, B. (2008): Notes for history of Chagas disease in Mexico [in Spanish]. Bol. Med. Hosp. Infant. Mex. 65, 57-79.

Villagrán, M. E., Martínez-Ibarra, J. A. and de Diego, J. A. (2011): Organic alterations and prevalence of Trypanosoma cruzi in opossums from western Mexico. Bol. Malar. Salud Ambient. 6, 87-88. 